\title{
Usage of antibiotics in the intensive care units of an academic tertiary-level hospital
}

\author{
Deanne Johnston $^{\mathrm{a}, \mathrm{b} *}$, Razeeya Khan ${ }^{\mathrm{a}}$, Jacqui Miot ${ }^{\mathrm{a}, \mathrm{b}}$ (D), Shirra Moch ${ }^{\mathrm{b}, \mathrm{c}}$, Yolande van Deventer ${ }^{\mathrm{a}}$ and Guy Richards ${ }^{\mathrm{d}, \mathrm{e}}$ \\ ${ }^{a}$ Department of Pharmacy and Pharmacology, University of the Witwatersrand, Johannesburg, South Africa \\ ${ }^{b}$ Critical Care Infection Collaboration, University of the Witwatersrand, Johannesburg, South Africa \\ ${ }^{c}$ Center for Health Science Education, University of the Witwatersrand, Johannesburg, South Africa \\ ${ }^{d}$ Division of Critical Care, University of the Witwatersrand, Johannesburg, South Africa \\ ${ }^{e}$ Department of Critical Care, Charlotte Maxeke Johannesburg Academic Hospital, Johannesburg, South Africa \\ *Corresponding author, email: deanne.johnston@wits.ac.za
}

Background: The post-antibiotic era is approaching fast as multidrug-resistant bacteria emerge and the antibiotic pipeline slows to a trickle. Antibiotic stewardship requires that antibiotics be used appropriately and as such this study reviewed the utilisation of antibiotics in five adult intensive care units (ICUs) at Charlotte Maxeke Johannesburg Academic Hospital.

Methods: A retrospective, cross-sectional record review of admissions to the Coronary, Cardiothoracic, Multidisciplinary, Neurology and Trauma ICUs was conducted over one month. Information from the ICU chart was captured on a modified version of the South African Antibiotic Stewardship Programme, Antibiotic Prescription Chart.

Results: A total of 204 files were reviewed with $55.2 \%$ of patients receiving antibiotics during admission. The three most frequently prescribed were amoxicillin clavulanate $(n=46)$, piperacillin/tazobactam $(n=32)$ and cefazolin $(n=28)$, while colistin was used on one occasion. The majority of antibiotics (84.9\%) were given for five days or less. Of concern however, of 35 instances where concurrent administration of antibiotics occurred, 8 had a similar spectrum and on 7 occasions the duration of antibiotic treatment was longer than 7 days. Cultures were ordered on 228 occasions. In patients receiving antibiotics $61.6 \%$ had cultures ordered; however, only $56.3 \%$ of these were taken before or on the day that antibiotics were started.

Conclusion: This study showed that generally the duration of antibiotic treatment was short, concurrent use of antibiotics was minimal and the use of a restricted formulary limited the use of specific antibiotics such as colistin. However, implementation of stewardship principles and ordering of appropriate cultures would assist in further improving appropriate use of antibiotics in the ICU setting.

Keywords: Antibiotic use, antibiotic stewardship, ICU, ultilisation

South Africa, like the rest of the world, is heading towards the post-antibiotic era with few new antibacterial agents expected in the next 10-15 years. There are increased reports of multidrug-resistant Enterobacteriaceae and the emergence of resistance to colistin, the last-line agent for Gram-negative bacteria in South Africa ${ }^{1}$ and abroad $^{2-5}$ has led to a call for global collaboration to improve prescribing practices.

In response, the South African National Department of Health has developed the Antimicrobial Resistance National Strategic Framework (2014-2024) whose vision it is to 'ensure the appropriate use of antimicrobials by health care professionals in all health establishments in South Africa, to conserve the efficacy of antimicrobials for optimal management of infections in human and animal health'. ${ }^{6}$ Wasserman et al., ${ }^{7}$ on behalf of the South African Antibiotic Stewardship Programme (SAASP), have also published 'A Pocket Guide to Antibiotic Prescribing for Adults in South Africa', which helps the prescriber to select an appropriate antibiotic as well as explaining the importance of appropriate cultures and the interpretation of these results.

Limited information is available on the use of antibiotics in the public sector in South Africa. A study conducted by Paruk et $a l^{8}$ reviewed antibiotic prescription practices and their relationship to outcomes in a number of ICUs in South Africa in both a public and private setting. The results were concerning as inappropriate choice of antibiotics and duration of treatment was common in both sectors and more so in the private sector. With this in mind we evaluated the use of antibiotics in the intensive care units (ICUs) at Charlotte Maxeke Johannesburg Academic Hospital (CMJAH).

\section{Methodology}

This was a retrospective, cross-sectional record review of admissions to the Coronary (CICU), Cardiothoracic (CTICU), Multidisciplinary (MICU), Neurology (NICU) and Trauma (TICU) Intensive Care Units of CMJAH. The period selected for review was from January 15, to February 15, 2016.

The aim of this study was to document and describe the use of antibiotics in ICUs at CMAJH. This would help to establish a baseline of utilisation prior to the introduction of a formal antimicrobial stewardship programme in the ICUs. To achieve this aim, the dose, duration and indication for use of all antibiotics administered in the study period were noted. These were analysed with regard to key antimicrobial stewardship principles, namely, the duration of treatment, concurrent use of antibiotics with a similar spectrum and if the prescribing of restricted antibiotics was empiric or culture directed.

A modified version of the SAASP Antibiotic Prescription Chart was used (with the authors' permission) as the data-collection 
tool. The antibiotics prescribed for adult patients (over the age of 18 years) admitted during the study period were included. Culture results, where available, were recorded for each patient.

\section{Study context}

A distinction between the various ICUs needs to be made to fully understand the data with reference to the types of conditions seen:

- Coronary ICU (CICU): (6 beds)

Patients are admitted for preauthorised procedures such as angiograms, and for acute cardiac events such as a myocardial infarction.

- Cardiothoracic ICU (CTICU): (12 beds)

The majority of admissions are for cardiothoracic and cardiac surgery, many of which are preauthorised procedures.

- Multidisciplinary ICU (MICU): (12 beds)

Admissions to this unit have either recently undergone complex surgery, are recent emergencies including respiratory distress, or patients with severe sepsis and multiple organ dysfunction with or without shock.

- Neurosurgery ICU (NICU): (8 beds)

Admissions to this unit are pre- or post-neurosurgical procedures.

- Trauma ICU (TICU): (9 beds)

Admissions are mostly for major trauma of any aetiology.

This is a diverse group of units with specific conditions being treated. It is therefore difficult to compare units in terms of the appropriateness of antibiotics used.

\section{Availability of antibiotics in the hospital}

The hospital is a public institutional facility and prescribers generally adhere to the Essential Medicine Lists. Therefore, not all antibiotics registered in South Africa are available as prescription choices.

The process of prescribing antibiotics in the hospital is summarised in Figure 1. Antibiotics are prescribed on the ICU chart by the attending physician. If the antibiotic is available from ward stock the patient is likely to get the dose immediately. Antibiotics not in ward stock are ordered from the pharmacy on a per patient basis providing there is appropriate documentation. Medications ordered for patients in the wards are issued by the pharmacy and recorded manually according to the ward.

Furthermore, the hospital has a list of specific antibiotics where the prescribing doctor needs permission from a member of the antimicrobial committee, which comprises microbiologists and infectious diseases specialists, to prescribe the medication, together with supporting documentation such as culture results, as represented in Figure 1.

\section{Data analysis}

Information from the data-capturing sheet was captured on Microsoft Excel ${ }^{\circledast}$ (2010) (Microsoft Corp, Redmond, WA, USA) and quantitatively assessed, making use of descriptive analyses as well as graphical representations and comparison tables. The following definitions applied.

\section{Concurrent antibiotics}

Antibiotics were defined as concurrent if two antibiotics or more were administered at the same time. This included the situation where the initial antibiotic was continued and one or more antibiotics were added to the prescription, or if two or more antibiotics were started at the same time.

\section{Culture results for restricted antibiotics}

Antibiotics used for more resistant micro-organisms and which have the highest degree of restriction in the hospital, specifically the carbapenems and colistin, were analysed in terms of culture results.

Treatment was categorised as empiric, culture-directed or empiric-directed, using the following definitions:

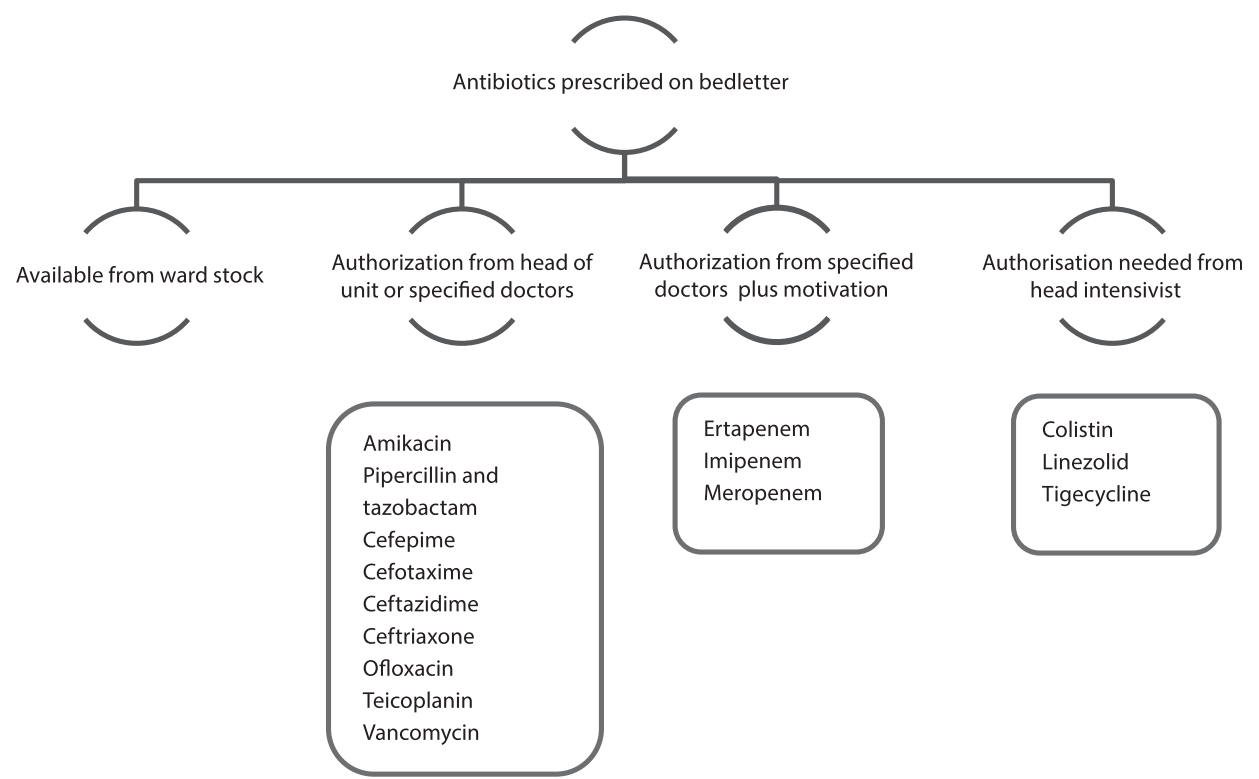

Figure 1: Process followed when ordering antibiotics. 
Empiric: no culture results were available;

Culture-directed: at the time of prescribing, a culture result indicated sensitivity to the specified antibiotic;

Empiric-directed: initially the antibiotic was prescribed empirically; however, during the course of treatment the culture result indicated sensitivity.

\section{Duration of antibiotic treatment}

The length of antibiotic treatment was calculated and grouped into one of four categories: less than 5 days, 5 to 7 days, 8 to 10 days and longer than 10 days.

\section{Ethical considerations}

Ethics approval for this study was obtained from the Human Research Ethics Committee of the University of the Witwatersrand (M141141). Each patient was assigned a unique study number and these together with the patient's record number, name and surname were kept in a password-protected data file accessible only to the researchers. The SAASP antibiotic prescription chart was modified to anonymise the data such that only the study number, age (years and months) and gender were captured.

\section{Results}

Records were reviewed in 204 adult patients across the 5 intensive care units of $\mathrm{CMJAH}$, with the highest numbers of patients seen in the MICU $(34.5 \% ; n=70)$ and $\mathrm{CICU}(29.6 \% ; n=60)$.

\section{Utilisation of antibiotics in the intensive care units}

Of the 28 parenteral antibiotics available for use in the hospital, $75 \%$ were used on at least one occasion. Cefotaxime, teicoplanin, linezolid and tigecycline were not used in any of the ICUs during the study dates. A few antibiotics, $30.8 \%$ of those available, were administered orally. These were amoxicillin, clarithromycin, co-trimoxazole and metronidazole.

The number of antibiotics that patients received during their stay in the ICU is summarised in Table 1. These values are inclusive of those administered concurrently (Table 2). Some $44.8 \%$

Table 1: Number of antibiotics that patients were prescribed sequentially or concurrently during their stay in the intensive care units

\begin{tabular}{|l|c|c|c|c|c|}
\hline & \multicolumn{4}{|c|}{ Percentage of patients receiving antibiotics } \\
\hline $\begin{array}{c}\text { Number } \\
\text { of ABs }\end{array}$ & $\begin{array}{c}\text { CICU } \\
(n=60)\end{array}$ & $\begin{array}{c}\text { CTICU } \\
(n=29)\end{array}$ & $\begin{array}{c}\text { MICU } \\
(n=70)\end{array}$ & $\begin{array}{c}\text { NICU } \\
(n=24)\end{array}$ & $\begin{array}{c}\text { TICU } \\
(n=21)\end{array}$ \\
\hline 0 & 71.7 & 31.0 & 35.7 & 29.2 & 33.3 \\
\hline 1 & 15.0 & 44.8 & 37.1 & 33.3 & 28.6 \\
\hline 2 & 10.0 & 10.3 & 11.4 & 29.2 & 19.0 \\
\hline 3 & 1.7 & 3.5 & 7.1 & 4.2 & 14.3 \\
\hline 4 & 1.7 & 3.5 & 4.3 & 4.2 & 4.8 \\
\hline 5 & 0 & 3.5 & 2.9 & 0 & 0 \\
\hline 6 & 0 & 0 & 1.4 & 0 & 0 \\
\hline 7 & 0 & 3.5 & 0 & 0 & 0 \\
\hline
\end{tabular}

ABs: antibiotics.

*Antibiotics where dose was increased/decreased were counted as one, provided there was no stop in treatment.

**Antibiotics where there was a change in route of administration were counted separately.

***If the same antibiotic was administered via two different routes simultaneously it was counted as two antibiotics.
Table 2: Brief case review of patients who received (a) seven and (b) six antibiotics while admitted to ICU

\section{Patient}

(a) Patient $X$

Length of stay in CTICU: 19 days

Age: 57 years

Sex: Male

Diagnosis: Initial admission for coronary artery bypass surgery. Patient later developed sepsis and pneumonia.

\section{Antibiotics:}

1. Vancomycin: $1 \mathrm{~g}$ IVI daily Empiric (3 doses, D1; D3 and D 6)

Levels measured on four occasions

2. Piperacillin/tazobactam: $18 \mathrm{~g}$ infusion Empiric (D3-D4) then restarted as Directed therapy on second admission to CTICU (Escherichia coli)

3. Imipenem: $1 \mathrm{~g}$ IVI stat followed by $500 \mathrm{mg} \mathrm{IVI}$ qid Empiric (5 doses, D4-D5)

4. Ertapenem: $1 \mathrm{~g}$ IVI daily (8 doses, D5-D12) Directed (Escherichia coli - blood culture)

5. Cefepime: $2 \mathrm{~g}$ IVI 8 hourly Empiric (5 doses, D5-D6) 6. Amikacin: $1 \mathrm{~g} \mathrm{IVI} \mathrm{stat} \mathrm{(1} \mathrm{dose,}$ D8)

7. Co-trimoxazole: $P O$ four tablets qid Directed (54 doses D15D19) Stenotrophomonas maltophilia - sputum Patient also received antifungals - Fluconazole

- Amphotericin B

(b) Patient $Y$

Length of stay in MICU: 8 days

Age: 33 years

Sex: Male

Diagnosis: Laparotomy for secondary peritonitis

\section{Antibiotics:}

1. Piperacillin/tazobactam: $2.25 \mathrm{mg}$ IVI tds Empiric (1 dose D1) patient admitted into MICU on this antibiotic

2. Ertapenem $1 \mathrm{~g}$ IVI bd Empiric (2 doses D1) then daily (2 doses D2-D3)

3. Erythromycin $250 \mathrm{mg}$ IVI bd (4 doses D1-D2) Prokinetic agent

4. Metronidazole $500 \mathrm{mg} \mathrm{IVI} \mathrm{tds}$ Empiric (13 doses D3-D8) C. Difficile

5. Metronidazole $400 \mathrm{mg} P O \mathrm{tds}$ Empiric (13 doses D4-D8) C. difficile

6. Piperacillin/tazobactam $4.5 \mathrm{mg}$ IVI tds Directed (15 doses D3D8)

Patient also received antifungals - Fluconazole

*NG: no growth.

Number of occasions biomarkers
were measured:
CRP: 6
Procalcitonin: 6
Culture results:
A. Fluid aspirate: $n=1$ -
Acinetobacter baumannii,
Enterococcus faecium
B. Tracheal aspirate: $n=1$ -
Acinetobacter baumannii
C. Urine: $n=1$ NG

( $n=91$ ) of patients did not receive antibiotics during their admission at all and the majority of these $(n=43)$ were admitted to the CICU. The MICU had the highest percentage use and of these $15.7 \%$ received three or more antibiotics (sequentially or concurrently) during their admission. The highest number of
Number of occasions biomarkers

were measured:

CRP: 22

Procalcitonin: 23

Culture results:

A. Blood: $n=5$ - NG (4); E. coli (1)

B. Catheter urine: $n=1 \mathrm{NG}$

C. Fluid aspirate: $n=1$

Escherichia coli

D. IV catheter tip: $n=2$

Coagulase negative Streptococcus Group G

Sputum: $n=3$ - Pseudomona aeruginosa; Escherichia coli:

Stenotrophomonas maltophilia

cal aspirate: Escherichia coli (2) 
Table 3: Concurrent administration of two or three antibiotics per ICU

\begin{tabular}{|c|c|c|c|c|}
\hline ICU & Antibiotic 1 & Antibiotic 2 & Antibiotic 3 & Comment \\
\hline \multirow[t]{4}{*}{$\mathrm{CICU}$} & Ceftriaxone (IV)* & Cloxacillin (IV) & & AB 1 \& 2: Overlapping spectrum but used for endocarditis \\
\hline & Amoxicillin (PO) & Clarithromycin (PO) & & Used for eradication of $\mathrm{H}$. pylori \\
\hline & Cloxacillin (IV) & $\begin{array}{l}\text { Piperacillin/ } \\
\text { tazobactam (IV) }\end{array}$ & & AB 1 \& 2: Overlapping spectrum \\
\hline & $\begin{array}{l}\text { Amoxicillin clavulanate } \\
\text { (IV) }\end{array}$ & $\begin{array}{l}\text { Piperacillin/ } \\
\text { tazobactam (IV) }\end{array}$ & & AB 1 \& 2: Overlapping spectrum \\
\hline \multirow[t]{6}{*}{ CTICU } & $\begin{array}{l}\text { Piperacillin/ } \\
\text { tazobactam (IV) }\end{array}$ & Vancomycin (IV) & & No overlap \\
\hline & Amikacin (IV) & Ertapenem (IV) & & $A B$ 1: Used empirically in $\mathrm{HAl}$ to broaden spectrum $A B 2$ \\
\hline & Ceftriaxone (IV) & Cloxacillin (IV) & & AB 1 \& 2: Overlapping spectrum: Infective endocarditis \\
\hline & Ertapenem (IV) & Vancomycin (IV) & & No overlap \\
\hline & Cefazolin (IV) & Vancomycin (IV) & & No overlap \\
\hline & Cefepime (IV) & Ertapenem (IV) & Vancomycin (IV) & $A B 1$ \& 2: Overlapping spectrum \\
\hline \multirow[t]{12}{*}{ MICU } & $\begin{array}{l}\text { Amoxicillin clavulanate } \\
\text { (IV) }\end{array}$ & Ertapenem (IV) & & $A B 1 \&$ 2: Overlapping spectrum \\
\hline & Ertapenem (IV) & Metronidazole (PO) & & $A B$ 2: used for possible Clostridium difficile infection \\
\hline & Metronidazole (PO) & Metronidazole (IV) & & $A B 1$ \& 2: Treatment Clostridium difficile infection \\
\hline & Clindamycin (IV) & $\begin{array}{l}\text { Amoxicillin clavulanate } \\
\text { (IV) }\end{array}$ & & $\begin{array}{l}A B \text { 1: 'Eagle effect' }{ }^{9} \text { used to inhibit ribosomal toxin production } \\
\text { in SSTI }\end{array}$ \\
\hline & Clindamycin (IV) & Cefepime (IV) & & $A B$ 1: added for treatment of skin and soft tissue infection \\
\hline & Ampicillin (IV) & Erythromycin (IV) & & AB 2: Prokinetic agent \\
\hline & Azithromycin (IV) & $\begin{array}{l}\text { Piperacillin/ } \\
\text { tazobactam (IV) }\end{array}$ & & $A B 1$ : used to inhibit quorum sensing especially pseudomonas \\
\hline & Azithromycin (IV) & Ertapenem (IV) & & $A B 1$ Quorum sensing effect: tertiary peritonitis \\
\hline & Azithromycin (IV) & $\begin{array}{l}\text { Amoxicillin clavulanate } \\
\text { (IV) }\end{array}$ & & $\begin{array}{l}\text { Used as immunomodulatory or to cover Legionella in severe } \\
\text { CAP }\end{array}$ \\
\hline & Clindamycin (IV) & Ciprofloxacin (IV) & & $A B$ 1: Anaerobic cover \\
\hline & Metronidazole $(\mathrm{PO})^{*}$ & Metronidazole (IV) & $\begin{array}{l}\text { Piperacillin/ } \\
\text { tazobactam (IV) }\end{array}$ & $A B 1$ \& 2: Clostridium difficile infection: local SOP \\
\hline & Amikacin (IV) & Ertapenem (IV) & Vancomycin (IV) & $A B$ 1: Used empirically in $\mathrm{HAl}$ to broaden spectrum of $A B 2$ \\
\hline \multirow[t]{2}{*}{ NICU } & $\begin{array}{l}\text { Amoxicillin clavulanate } \\
\text { (IV) }\end{array}$ & Metronidazole (IV) & & $\begin{array}{l}\text { AB 2: Probably overlapping spectra but also used for } \\
\text { Clostridium difficile infection }\end{array}$ \\
\hline & Ceftriaxone (IV) & Metronidazole (PO) & & No overlap \\
\hline \multirow[t]{9}{*}{ TICU } & Azithromycin (IV) & Imipenem (IV) & & $A B 1$ Quorum sensing effect: tertiary peritonitis \\
\hline & Imipenem (IV) & Vancomycin (IV) & & No overlap \\
\hline & Azithromycin (IV) & Ertapenem (IV) & & $A B 1$ Quorum sensing effect: tertiary peritonitis \\
\hline & Azithromycin (IV) & Cefazolin (IV) & & Cannot explain this \\
\hline & Clindamycin (IV) & $\begin{array}{l}\text { Piperacillin/ } \\
\text { tazobactam (IV) }\end{array}$ & & $\begin{array}{l}\text { AB 1: 'Eagle effect' }{ }^{9} \text { used to inhibit ribosomal toxin production } \\
\text { in SSTI }\end{array}$ \\
\hline & Imipenem (IV) & $\begin{array}{l}\text { Piperacillin/ } \\
\text { tazobactam (IV) }\end{array}$ & & $A B 1 \&$ 2: Overlapping spectrum \\
\hline & Imipenem (IV) & Ciprofloxacin (IV) & & Overlap but may broaden spectrum in $\mathrm{HAI}$ \\
\hline & Imipenem (IV) & Erythromycin (IV) & & $A B$ 2: Prokinetic agent \\
\hline & Azithromycin (IV) & $\begin{array}{l}\text { Amoxicillin clavulanate } \\
\text { (IV) }\end{array}$ & $\begin{array}{l}\text { Piperacillin/ } \\
\text { tazobactam (IV) }\end{array}$ & $\begin{array}{l}A B 1 \& 2: \text { Used as immunomodulatory or to cover Legionella in } \\
\text { severe CAP }\end{array}$ \\
\hline
\end{tabular}

*This combination of antibiotics was used on two occasions.

CAP: Community-acquired pneumonia. HAI: Hospital-acquired infections. SOP: Standard operating procedure. STTI: Skin and soft tissue infection.

antibiotics ( $n=7$, again sequentially or concurrently) administered to a patient was in the CTICU.

A brief case description for the two patients from the CTICU and MICU who received seven and six antibiotics respectively is summarised in Table 2.

The most frequently prescribed antibiotics were amoxicillin clavulanate $(21.9 \%)$, piperacillin/tazobactam (21.9\%), cefazolin (13.3\%) and ertapenem (9.0\%). Four antibiotics, amoxicillin
(PO), clarithromycin (PO), colistin (IV) and meropenem (IV), were used on one occasion each.

\section{Concurrent administration of antibiotics}

The number of antibiotics received during the ICU admissions (Table 1) was reviewed in conjunction with the number of antibiotics given concurrently (Table 3 ). On 30 occasions two antibiotics were administered concurrently and on 5 occasions three antibiotics were given. 
Combinations of antibiotics including macrolides (azithromycin and erythromycin) were used on a few occasions in the MICU and TICU and combinations including penicillins, cephalosporins and/or carbapenems were seen on eight occasions in the $\mathrm{CICU}$, CTICU and TICU. Metronidazole was administered both orally and intravenously in three patients while admitted to the MICU.

\section{Daily dose of antibiotics}

The prescribed daily dose of the antibiotics administered was compared with the defined daily dose (DDD). A DDD could not be calculated for all the antibiotics used as there were no records of the number of doses dispensed but then returned to the pharmacy. Therefore, a comparison was made between the prescribed daily dose and the DDD. The majority of the prescribed daily doses exceeded the DDD: amoxicillin (PO); cefazolin (IV); cefepime (IV); ceftriaxone (IV); ciprofloxacin (IV); clindamycin (IV); cloxacillin (IV); colistin (IV); ertapenem (IV); gentamicin (IV); imipenem (IV); metronidazole (IV) and piperacillin/ tazobactam (IV). Doses of ceftazidime (IV) and meropenem (IV) were lower than the DDD.

\section{Missed doses of antibiotics}

A total of 32 doses were missed according to patient files. Stock shortage was noted on three of these occasions. This was highest in the TICU $(n=12)$ where one patient missed six doses.

\section{Duration of antibiotic treatment}

The majority of antibiotics (84.9\%) were given for less than 5 days and numerous antibiotics were given for 24 hours or less. This is understandable for amoxicillin/clavulanic acid and cefazolin, both of which are used prophylactically in surgical patients, but amikacin, azithromycin, ceftriaxone, cotrimoxazole, ertapenem, erythromycin, gentamicin, imipenem, metronidazole (PO and IV), piperacillin/tazobactam and vancomycin were given for a day or less in at least one patient.

The duration of antibiotic treatment was seven days or longer in $3.77 \%(n=8)$ of antibiotics prescribed. Ertapenem (IV) was administered for eight days on two occasions, once in the CTICU and once in the NICU. In the MICU piperacillin/tazobactam was prescribed to two different patients for eight days. Ceftriaxone was used for nine days in one patient in the NICU. On two occasions the duration of imipenem treatment exceeded eight days in the TICU.

To determine if the duration of antibiotic therapy was appropriate, it is necessary to refer to the diagnosis. This was not possible as in most instances the original admission diagnosis was recorded by the nurse on the ICU chart without subsequent modification. However, very few therapies exceeded 7-8 days, which is generally an appropriate duration for HAI.

\section{Cultures}

A total of 228 cultures were sent to the laboratory. The majority of these were from the MICU $(41.7 \% ; n=95)$ and only seven cultures were requested from NICU. In the 112 patients who received antibiotics while admitted to the ICUs, $61.6 \%(n=69)$ had cultures done (Figure 2). This varied considerably between the MICU (82.2\%) and the CICU (17.7\%). Furthermore, it was determined that cultures were taken either before or on the day that the antibiotic was started in $56.3 \%$ of cases. However, this was far higher in the NICU (75\%) and MICU (70\%).

\section{Indication for use of restricted antibiotics}

Culture results were reviewed in patients receiving either a carbapenem or colistin. It was found that $70 \%$ of the carbapenems were prescribed empirically, $23.3 \%$ were directed and $6.7 \%$ were empiric-directed (Figure 3). The vast majority of $\mathrm{HAI}$ at CMJAH are due to extended spectrum beta lactamase (ESBL) producing enterobacteriaceae that are also resistant to fluoroquinolones and piperacillin/tazobactam.

Colistin was administered only once during the study period to a patient in the MICU where the blood culture revealed a carbapenem-resistant Enterobacter aerogenes.

\section{Discussion}

Antibiotics are frequently prescribed in the intensive care setting $;^{10}$ however, very few studies have documented their use in public hospitals. ${ }^{8}$ Across the five ICUs, $55.2 \%$ of patients received antibiotics. Chunnilall et al. ${ }^{11}$ reported that $28.8 \%$ of patients in a private ICU in KwaZulu-Natal received antibiotics while Paruk et al. ${ }^{8}$ reported a $73.4 \%$ antibiotic prescription rate when they reviewed antibiotic prescribing practices across the public and private sectors.

\section{Formulary restrictions to reduce antibiotics usage}

Use of a restricted formulary has reduced the use of specific antibiotics in the hospital and this along with a cost reduction has previously been shown. ${ }^{12-14}$ It is difficult, however, to ensure adherence to such a system without adequate ongoing surveillance of prescribing habits, and there is the added concern that such restrictions could promote the emergence of resistance to other antibiotics. ${ }^{12,15}$ Further analysis is necessary to determine if there has been increased emergence of resistance to the antibiotics that are prescribed more frequently in these units. The pharmacy is the gate-keeper, especially in ensuring that the correct processes have been followed before issuing of the restricted items. This is, however, where their role at this study site ends.

The restricted formulary has reduced the use of carbapenems and colistin although ertapenem is used most frequently when empiric treatment is initiated for HAl. Use of meropenem in the hospital is limited to neonates and patients at risk of, or currently experiencing, seizures because carbapenems are awarded as a class on the national tender.

Colistin is reserved for patients who have an infection at a usually sterile site that is resistant to other agents. In our study, colistin was used in line with the South African Colistin Guidelines 2016, ${ }^{16}$ which includes a loading dose of $12 \mathrm{mu}$ followed by 3 mu eight hourly.

\section{Concurrent administration of antibiotics}

It is difficult to assess the appropriateness of concurrent use of antibiotics retrospectively. It would have been useful to consult with the prescribing doctors but as this was a retrospective study it was not possible. Probable explanations have been provided in Table 2 . Three scenarios should be considered:

Patients presenting with Clostridium difficile infection are given metronidazole orally and intravenously concurrently until the diarrhoea resolves. Thereafter the patient is continued on the oral dosage form for a total of 14 days. This combination is the standard operating procedure at CMJAH and the regimen was given on three occasions in the MICU. However, this regimen is not 


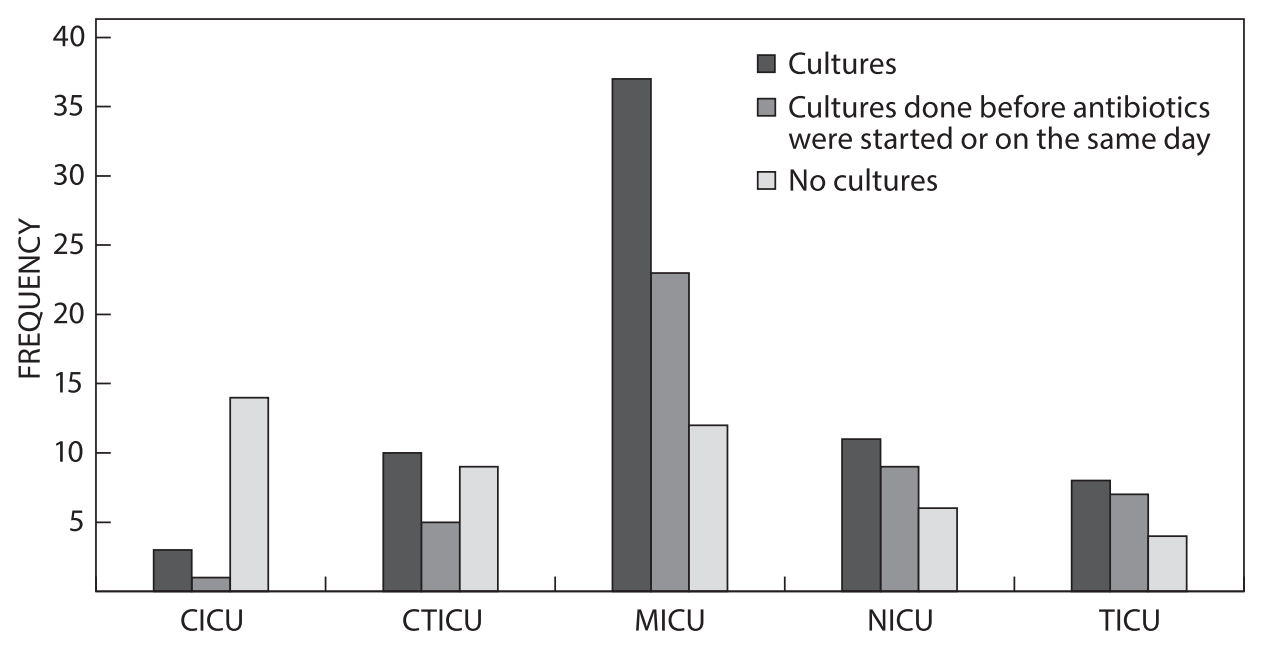

Figure 2: Number of patients on antibiotics who had cultures performed as per ICU.

recommended by the South African Standard Treatment Guidelines and Essential Medicines List or international guidelines. Metronidazole is a concentration-dependent antibiotic and as such there is a theoretical rationale for its use at this dose. We are in the process of evaluating the relapse rate of Clostridium difficile on this regimen as anecdotally it seems to be extremely rare.

Azithromycin and erythromycin were frequently administered concurrently with other antibiotics in the MICU and TICU; however, this was not for their antibacterial properties. Erythromycin is administered intravenously as a prokinetic agent ${ }^{17}$ in patients with ileus and azithromycin for its effect on quorum sensing and specifically on biofilm formation. $^{18}$

Amikacin was used in conjunction with ertapenem on two occasions. Amikacin is used to broaden the cover when treating $\mathrm{HAl}$ empirically. ${ }^{19}$

In comparison with the study in the ICUs of both the public and private sectors mentioned previously, in which up to 10 antibiotics were given simultaneously (attributed to previous antibiotics not having been stopped when new ones were prescribed), ${ }_{1}^{8}$ in this study no more than three antibiotics were administered concurrently. However, some of these combinations did seem to provide either overlapping spectra or redundant cover.

\section{Daily dose of antibiotics}

Many of the prescribed daily doses exceeded the DDD, which were most likely prescribed according to pharmacokinetic principles. The rationale for higher doses is related to the lower than expected drug concentrations in critically ill patients. ${ }^{20}$

Plasma concentrations of hydrophilic antibiotics may be decreased owing to the increased volume of distribution due to capillary leak, the reduced albumin (for highly protein bound drugs) and fluid overload. In addition augmented renal clearance (ARC) due to increased cardiac output from use of inotropes, and renal replacement therapy ${ }^{21}$ may contribute. For optimal patient management the use of the correct antibiotic, dose and method of administration is essential. Dose adjustment directed towards weight, renal function and the pharmacokinetic properties of each drug, particularly in the critically ill patient, is an essential component of stewardship. $^{22}$ Doses lower than the DDD were seen in patients in renal failure unless they were on continuous renal replacement therapy, in which case conventional doses were prescribed.

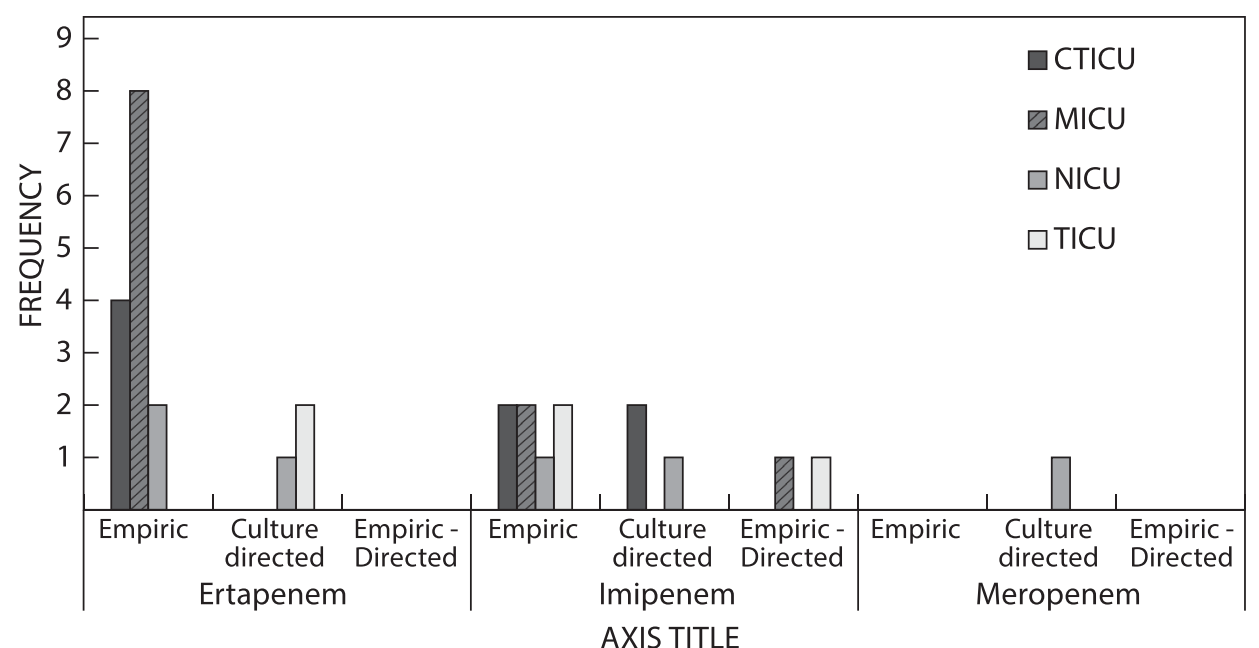

Figure 3: Number of prescriptions per ICU for carbapenems according to culture result. 


\section{Duration of antibiotic treatment}

In a study mentioned previously that was conducted in both public and private sector ICUs in South Africa, the duration of antibiotic treatment was inappropriate in $72 \%$ of patients. ${ }^{8}$ In this study the duration ranged from 0.25 to 10.3 days with the majority $(n=180)$ receiving 5 days' worth or less. Some antibiotics were administered empirically for less than 24 hours. Other than those given for surgical prophylaxis, some of these short courses may be accounted for by the fact that antibiotics are frequently initiated by junior doctors and subsequently reviewed by senior clinicians who change to a more appropriate antibiotic. Lawrence and Kollef ${ }^{23}$ concluded that shorter duration is in fact the goal; however, it should be individualised according to the organism, the presence of immunosuppression and the resolution of biomarkers.

\section{Cultures}

Cultures were not ordered consistently across all units. Not all patients receiving antibiotics had cultures taken and cultures were often performed after antibiotics were started. Some of these were when the patient received prophylactic antibiotics for 24 hours postoperatively and as such cultures were not performed. However, in many cases cultures were not taken before the antibiotic was started. This is a serious breach of antibiotic stewardship principles as interpretation of the antibiogram is pivotal to both the selection of an appropriate agent and to de-escalation of therapy. ${ }^{7}$

\section{Study limitations and further recommendations}

The design of the SAASP antibiotic prescription chart is such that it links the antibiotic to the infection. This could be used to determine if the antibiotic that was prescribed was appropriate. This correlation was not possible in this study, however, as this is not requested on the ICU charts. In reviewing patients' files retrospectively, it is also not possible to consult with both doctors and nurses to confirm the information and discrepancies found. The study period was within the summer months, and it is not possible to know the extent to which seasonal variation impacts on antibiotic usage.

An ongoing longitudinal, prospective study of the impact of a hospital-wide intervention programme, designed to improve antibiotic use, would add to the current data and assist in furthering the antimicrobial stewardship programme. Part of this intervention has been the introduction of an antimicrobial prescription chart, based on that promoted by SAASP.

An electronic system for dispensing medication and monitoring of prescribed antibiotics is essential. Thursky et al. ${ }^{24}$ found that the implementation of a computerised system, incorporating the microbiology and a decision system, assisted in reducing the use of unnecessary broad-spectrum agents. Similarly, obtaining timeous and appropriate specimens prior to initiating antibiotic treatment is critical ${ }^{20}$ in ensuring the correct antibiotic choice and facilitating de-escalation. In CMJAH future studies need to be cognisant of these fundamental principles.

A multidisciplinary antimicrobial team to enforce and measure the effectiveness of a restricted formulary as well as the implementation of further stewardship practices is also critical to effective stewardship. Brink et $a .^{25}$ have commented on the role that nurses and pharmacists play in antibiotic stewardship and called for a collaborative effort in the fight against the inappropriate use of antibiotics.

\section{Conclusion}

This study documented the use of antibiotics in CMJAH in relation to antibiotic stewardship principles within a setting where a formulary is applied, and certain antibiotics are restricted. The overall duration of antibiotic treatment was short and concurrent use of redundant agents was minimal. The doses of antibiotics mostly exceeded the DDD while doses lower than the DDD were mainly used in renal failure. An important intervention, however, is to ensure that cultures are more frequently performed. Colistin was seldom used and when it was, it was dosed according to current guidelines. More stringent controls and the implementation of stewardship principles are still necessary to further reduce the inappropriate use of antibiotics.

Disclosure statement - No conflict of interest was reported by the authors.

Funding - The research was funded by the researchers.

\section{ORCID}

Jacqui Miot (i) http://orcid.org/0000-0001-7070-3826

\section{References}

1. Brink AJ, Coetzee J, Corcoran C, et al. Emergence of OXA-48 and OXA181 Carbapenemases among Enterobacteriaceae in South Africa and Evidence of In Vivo selection of colistin resistance as a consequence of selective decontamination of the gastrointestinal tract. J Clin Microbiol 2013;51(1):369-72. Available from: http://jcm.asm.org/ content/51/1/369.full

2. Mammina C, Bonura C, Bernardo D, et al. Ongoing spread of colistinresistant Klebsiella pneumoniae in different wards of an acute general hospital, Italy, June to December 2011. Eur Commun Dis Bull. 2012;17(33)ii:20248. Available from: http://www.eurosurve illance.org/ViewArticle.aspx?Articleld=20248

3. Halaby T, al Naiemi N, Kluytmans J, et al. Emergence of colistin resistance in Enterobacteriaceae after the introduction of selective digestive tract decontamination in an intensive care unit. Antimicrob Agents Chemother. 2013;57(7):3224-9. Available from: https://www. ncbi.nlm.nih.gov/pmc/articles/PMC3697390/

4. Del Franco M, Paone L, Novati R, et al. Molecular epidemiology of carbapenem resistant Enterobacteriaceae in Valle d'Aosta region, Italy, shows the emergence of KPC-2 producing Klebsiella pneumoniae clonal complex 101 (ST101 and ST1789). BMC Microbiol. 2015;15 (1):260. Available from: https://bmcmicrobiol.biomedcentral.com/ articles/10.1186/s12866-015-0597-z

5. Jayol A, Poirel $L$, Dortet $L$, et al. National survey of colistin resistance among carbapenemase-producing Enterobacteriaceae and outbreak caused by colistin-resistant OXA-48-producing Klebsiella pneumoniae, France, 2014. Eur Commun Dis Bull. 2016;21(37). Available from: http://www.eurosurveillance.org/ViewArticle.aspx?Articleld= 22579

6. National Department of Health. A practical guide to antimicrobial stewardship in South Africa. [cited 2017 June 14]. Available from: http://www.health.gov.za/index.php/antimicrobial-resistance?downl oad=1493:ams-guidelines-ndoh-draft-v1-0.

7. Wasserman S, Boyles T, Mendelsohn M. A pocket guide to antibiotic prescribing for adults in South Africa. First published 2014 () Sean Wasserman, Tom Boyles, Marc Mendelson. [cited 2017 August 4]. Available from: http://www.fidssa.co.za/Content/Documents/SAA SP_Antibiotic_Guidelines_2015.pdf

8. Paruk F, Richards G, Scribante J, et al. Antibiotic prescription practices and their relationship to outcome in South Africa: findings of the prevalence of infection in South Africa intensive care units (PISA) study. S Afr Med J. 2012;102(7):613-6. Available from: http://www. samj.org.za/index.php/samj/article/view/5833/4292

9. Stevens DL, Gibbons AE, Bergstrom R, et al. The Eagle effect revisited: efficacy of clindamycin, erythromycin, and penicillin in the treatment of streptococcal myositis. J Infect Dis. 1988 Jul 1;158(1):23-8. 
Available from: https://academic.oup.com/jid/article-abstract/158/1/ 23/786909

10. Badar VA, Navale SB. Study of prescribing pattern of antimicrobial agents in medicine intensive care unit of a teaching hospital in central India. J Assoc Physicians India. 2012;60:20-3. Available from: http://www.japi.org/april_2012/04_oa_study_of_prescribing.pdf

11. Chunnilall D, Peer AK, Naidoo I, et al. An evaluation of antibiotic prescribing patterns in adult intensive care units in a private hospital in KwaZulu-Natal. S Afr J Infect Dis. 2015;30:17-22. Available from: http://www.sajei.co.za/index.php/SAJEl/article/view/620

12. Rahal JJ, Urban C, Horn D, et al. Class restriction of cephalosporin use to control total cephalosporin resistance in nosocomial Klebsiella. JAMA. 1998;280(14):1233-1237. Available from: http://jamanetwork. com/journals/jama/fullarticle/188047

13. Bassetti M, Di Biagio A, Rebesco B, et al. The effect of formulary restriction in the use of antibiotics in an Italian hospital. Eur J Clin Pharmacol. 2001;57(6-7):529-34. Available from: https://link. springer.com/content/pdf/10.1007/s002280100338.pdf

14. Dellit TH, Owens RC, McGowan JE Jr., et al. Infectious diseases society of America and the society for healthcare epidemiology of America guidelines for developing an institutional program to enhance antimicrobial stewardship. Clin Infect Dis. 2007;44(2):159-77. Available from: http://www.idsociety.org/uploadedFiles/IDSA/Guidelines-Patie nt_Care/PDF_Library/Antimicrobial\%20Stewardship.pdf

15. Meyer KS, Urban C, Eagan JA, et al. Nosocomial outbreak of Klebsiella infection resistant to late-generation Cephalosporins. Ann Intern Med. 1993;119(5):353-358. Available from: http://www.ajicjournal. org/article/0196-6553(95)90013-6/abstract

16. Labuschagne Q, Shellack N, Gous A, et al. COLISTIN: adult and paediatric guideline for South Africa, 2016. S Afr J Infect Dis. 2016;31(1):3-7. Available from: http://www.tandfonline.com/doi/pdf/10.1080/2312 0053.2016.1144285

17. Doherty WL, Winter B. Prokinetic agents in critical care. Crit Care. 2003;7(3):206-8. Available from: https://www.ncbi.nlm.nih.gov/pmc/ articles/PMC270665/
18. Kaplan JB. Antibiotic-induced biofilm formation. Int J Artif Organs. 2011;34(9):737-51. Available from: http://www.artificial-organs.com/ article/antibiotic-induced-biofilm-formation-ijao-d-11-00105

19. Giamarellou H. Aminoglycosides plus beta-lactams against gramnegative organisms: evaluation of in vitro synergy and chemical interactions. Am J. Med. 1986;80(6):126-37. Available from: http:// www.sciencedirect.com/science/article/pii/0002934386904900

20. Richards GA, Joubert IA, Brink AJ. Optimizing the administration of antibiotics in critically ill patients. S Afr Med J. 2015;105(5):419. Available from: http://www.scielo.org.za/scielo.php?script=sci_artte xt\&pid=S0256-95742015000500035

21. Luyt C-E, Bréchot N, Trouillet J-L, et al. Antibiotic stewardship in the intensive care unit. Crit Care. 2014;18:215-480. Available from: https://ccforum.biomedcentral.com/articles/10.1186/s13054-0140480-6

22. Kumar A, Singh NP. Antimicrobial dosing in critically ill patients with sepsis-induced acute kidney injury. Indian J Crit Care Med. 2015;19 (2):99-108. Available from: https://www.ncbi.nlm.nih.gov/pmc/ articles/PMC4339912/

23. Lawrence $\mathrm{KL}$, Kollef $\mathrm{MH}$. Antimicrobial stewardship in the intensive care unit advances and obstacles. Am J Respir Crit Care Med. 2009;179(6):434-38. Available from: http://www.atsjournals.org/doi/ full/10.1164/rccm.200809-1394CP

24. Thursky KA, Buising KL, Bak N, et al. Reduction of broad-spectrum antibiotic use with computerized decision support in an intensive care unit. Int J Qual Health Care. 2006;18(3):224-31. Available from: https://academic.oup.com/intqhc/article/18/3/224/1786346/ Reduction-of-broad-spectrum-antibiotic-use-with

25. Brink AJ, van den Bergh $D$, Mendelson $M$, et al. Passing the baton to pharmacists and nurses: new models of antibiotic stewardship for South Africa?. S Afr Med J. 2016;106(10):947-948. Available from: http://www.samj.org.za/index.php/samj/article/view/11448

Received: 30-08-2017 Accepted: 28-05-2018 